\title{
Psychotic Symptoms in an Urban General Medicine Practice
}

Mark Olfson, M.D., M.P.H.

Roberto Lewis-Fernández, M.D.

Myrna M. Weissman, Ph.D.

Adriana Feder, M.D.

Marc J. Gameroff, M.A.

Daniel Pilowsky, M.D.

Milton Fuentes, Psy.D.
Objective: The authors' goals were to estimate the prevalence of psychotic symptoms among adults attending an urban general medical practice that serves a low-income population and to describe the mental health, social and occupational functioning, and mental health treatment of these patients.

Method: Data were drawn from a recent study of adult primary care patients $(\mathrm{N}=$ $1,005)$ in a large, urban, university-affiliated general medicine practice. During a medical visit, patients completed the psychotic disorders section of the Mini International Neuropsychiatric Interview, the Primary Care Evaluation of Mental Disorders, a drug use disorders screen, the Sheehan Disability Scale, and a questionnaire that probed demographic characteristics, health status, and mental health treatment.

Results: Two hundred ten $(20.9 \%)$ patients reported one or more psychotic symptoms, most commonly auditory hallucinations. There was an inverse correlation between family income and the prevalence of psychotic symptoms and a positive association between prevalence and Hispanic ethnicity. Compared with patients without psychotic symptoms, patients with psychotic symptoms were significantly more likely to have major depression $(42.4 \%$ versus $12.6 \%$ ), panic disorder (24.8\% versus $4.0 \%)$, generalized anxiety disorder (38.6\% versus $8.4 \%$ ), and alcohol use disorder (12.9\% versus $5.0 \%$ ). They were also more likely to report current suicidal ideation (20.0\% versus $3.5 \%$ ), recent work loss (55.0\% versus $35.6 \%$ ), and marital distress (28.6\% versus $13.0 \%$ ). Approximately one-half of the patients with psychotic symptoms (47.6\%) had taken a prescribed psychotropic medication during the last month.

Conclusions: Psychotic symptoms were highly prevalent in this primary care practice. These patients were at risk for several common mental disorders and often reported impaired work and social functioning. Future research should clarify the extent to which psychotic symptom reports among Hispanic patients are affected by culturally patterned idioms of distress. Clinicians who work in primary care practices that serve low-income patient populations should routinely inquire about psychotic symptoms.
$\mathrm{P}$ orders (e.g., schizophrenia, bipolar disorder, major depression with psychotic features, and substance-induced psychotic disorders) and general medical conditions (e.g., thyrotoxicosis, multiple sclerosis, acute intermittent porphyria, and brain tumors). In addition, a significant proportion of the general population reports a history of psychotic symptoms in the apparent absence of well-defined psychiatric disorder or general medical illness (1-3).

Uncertainty surrounds the clinical and nosologic status of psychotic symptoms identified with screening instruments or structured diagnostic assessments (4). Without information concerning the context of the symptoms, it may be difficult to distinguish clinically significant psychotic symptoms from momentary cognitive misperceptions, culturally sanctioned religious experiences, or respondent misunderstandings that do not represent mental illness. However, individuals who endorse psychotic symp- toms on screening interviews have been found to have higher rates of psychiatric hospitalization and several mental disorders $(2,5)$. Especially within cross-cultural samples, additional information may be required to distinguish true psychotic states from culturally patterned "idioms of distress.” These expressions of mental and emotional suffering, which arise in response to adversity, may be based on dissociative processes and supernatural illness attributions and are easily confused with psychotic illness (6).

Very little attention has been devoted to understanding the occurrence and clinical significance of psychotic symptoms among primary care patients. Although considerable effort has been devoted to characterizing mood, anxiety, and substance-related disorders in primary care populations (7-9), research on psychosis in primary care remains scarce $(5,10,11)$. Two studies suggest that schizophrenia and related disorders are rare in primary care clin- 
ics $(0.4 \%$ to $1.0 \%)(10,11)$. However, psychotic symptoms may be far more common (5). At one internal medicine group practice serving a middle-class patient population, $3.7 \%$ of adult patients reported experiencing one or more psychotic symptoms. These symptoms were strongly associated with functional impairment as well as mood, anxiety, or substance-related disorders (5).

Community surveys consistently demonstrate an inverse relationship between income and risk of mental disorders $(12,13)$. Moreover, psychotic disorders occur disproportionately among people who are poor, are unemployed, or have little formal education $(1,14)$. Some evidence further indicates that psychosis is more common in urban than rural populations (15-17). For these reasons, we examined the frequency, type, and distribution of psychotic symptoms in an urban general medicine practice serving a low-income patient population. We hypothesized that psychotic symptoms would be highly prevalent in this setting.

Most of the patients served in this general medicine practice are immigrants from Latin America, particularly from the Hispanic Caribbean. Idioms of distress associated with spiritual beliefs and practices common in Caribbean cultures may complicate the interpretation of selected psychotic symptoms in these cultures. For example, reports of auditory, visual, and tactile misperceptions in the context of affective disorders have resulted in a misdiagnosis of schizophrenia or other psychotic illness in epidemiological (18) and clinical $(19,20)$ samples of Hispanics. Belief in the "evil eye" and the disembodied power of envy as explanations for misfortune (21), as well as santería and espiritista practices of spirit possession and definitions of distress as spirit intrusion $(22,23)$, may prompt individuals to endorse items intended to detect paranoid delusions, thought insertion, and delusions of control, respectively. To help distinguish the extent to which items from a psychotic symptom screening interview represent normal religious experiences as opposed to impaired psychological states, we examined the relationship between self-reported psychotic symptoms and work, social, and family functioning. We also explored the relationship between endorsing psychotic symptoms and psychotic disorders to help clarify the extent to which reported symptoms may reflect nonspecific idioms of distress rather than clear evidence of psychotic illness.

\section{Method}

This study was conducted at the Associates in Internal Medicine, the faculty and resident group practice of the Division of General Medicine at the College of Physicians and Surgeons of Columbia University. The practice serves approximately 18,000 patients each year from the surrounding northern Manhattan community. The patients are predominantly low-income immigrants from the Dominican Republic and Puerto Rico. All forms were translated from English to Spanish and back-translated to identify and correct translational inconsistencies. This study was approved by the relevant institutional review boards before the start of data collection.

\section{Protocol and Patient Recruitment}

A systematic probability waiting room sample of adult primary care patients with scheduled appointments were invited to participate in the study. Eligible patients were between 18 and 70 years of age, had made at least one previous visit to the practice, could speak and understand Spanish or English, and were scheduled for face-to-face contact with their primary care physician. Patients were excluded from the study if their current general health status prohibited completion of the survey forms. Patients who were judged actively suicidal or homicidal were also excluded from the study, and appropriate emergency care was provided. Only patients who provided written informed consent after having had the study fully explained to them were enrolled.

A total of 3,427 patients were prescreened; 1,264 met study eligibility criteria. The most common reasons for exclusion were patient age $(\mathrm{N}=940,43.5 \%)$ and not having a scheduled visit with a primary care physician $(\mathrm{N}=672,31.1 \%)$. Of the 1,264 who met eligibility criteria, $1,005(79.5 \%)$ consented to participate in the study.

A systematic one-third random subsample of the first 813 participating patients $(\mathrm{N}=271)$ was selected for the diagnostic substudy described later in this article. The selected and nonselected patients did not differ significantly with respect to mean age, gender distribution, racial/ethnic background, family income, or educational attainment. Most of the selected patients agreed to participate in the substudy $(\mathrm{N}=223,82.3 \%)$. Those who agreed to participate did not significantly differ from those who refused with respect to mean age, gender, racial/ethnic background, or family income. However, patients who participated in the substudy had a higher level of education than those who were selected but refused to participate (Mann-Whitney $\mathrm{z}=-2.8$, $\mathrm{p}=$ 0.005).

\section{Diagnostic Substudy}

The patients in the random sample $(\mathrm{N}=223)$ were assessed within 1 week (mean $=4$ days) of the index medical visit. These patients were assessed with the psychotic disorders section of the Composite International Diagnostic Interview (24) $(\mathrm{N}=211)$ and the borderline personality disorder section of the Structured Clinical Interview for DSM-IV Axis II Personality Disorders (SCID-II) (25) $(\mathrm{N}=218)$. The diagnostic interviews were conducted by bilingual and bicultural master's and doctoral level mental health professionals who were blind to the results of the assessment completed in the clinic waiting room. They received a 4-day training course on the administration of the Composite International Diagnostic Interview and SCID-II that included monitored interviews and expert reevaluation.

\section{Sociodemographic and Clinical Assessment}

At study intake, the full sample of patients $(\mathrm{N}=1,005) \mathrm{com}-$ pleted a patient history form to assess their age, sex, race/ethnicity, marital status, family income, educational achievement, and self-rated overall health (excellent, very good, good, fair, or poor). The history form also included current major depressive disorder, current panic disorder, current generalized anxiety disorder, and past year alcohol sections of the Patient Health Questionnaire for the Primary Care Evaluation of Mental Disorders (DSM-IV version) (26). A drug use disorder module, patterned after the Patient Health Questionnaire for the Primary Care Evaluation of Mental Disorders alcohol use disorder module, was also administered to the patients.

Disability was measured with the 10-point self-rated family life/home responsibilities and social life items from the Sheehan Disability Scale $(27,28)(0=$ none, $1-3=$ mild, $4-6=$ moderate, $7-9=$ 
marked, $10=$ extreme). Three subjects did not answer any of the Sheehan Disability Scale items. Because only 199 (19.8\%) of the patients were gainfully employed, the Sheehan Disability Scale work item was not used in the following analyses. Modified items from the Social Adjustment Scale (29) were used to evaluate how patients were getting along with family members (very well, well, poorly, or very poorly). Ratings of poorly or very poorly were considered indicative of family distress. Work loss (yes or no) was assessed by asking whether during the past month patients had lost one or more days of work or school or had been unable to do housework or other regular activities. Self-perceived emotional health was measured on a 5-point Likert response scale: poor, fair, good, very good, or excellent. Patients were also asked a series of questions concerning professional treatment that they may have received for an emotional or mental problem.

\section{Psychotic Symptoms}

To assess psychotic symptoms, the Mini International Neuropsychiatric Interview (30), which includes 10 questions related to psychotic symptoms, was administered by a bilingual lay interviewer. The questions probe lifetime occurrence of paranoid delusions, delusions of control, delusions of reference, thought insertion, thought broadcasting, and auditory and visual hallucinations. Positive responses regarding any of the symptoms are followed up with a question to determine whether the symptoms are currently present. In the following analyses, patients were classified as having psychotic symptoms if they currently had at least one of the 10 psychotic symptoms.

\section{Statistical Methods}

Comparisons between patients with and without psychotic symptoms on categorical variables were made with the chisquare test, except when an expected cell size fell below 5 , in which case Fisher's exact test was used. Student's t test was used for comparisons involving continuous variables, and the MannWhitney test was used for comparisons involving ordered categories. Multiple linear regression controlling for age, sex, race, DSMIV disorders (defined by using the Patient Health Questionnaire for the Primary Care Evaluation of Mental Disorders), and drug use disorders was used to model the association between psychotic symptoms and Sheehan Disability Scale scores. Logistic regressions controlling for various covariates were used to model associations between psychotic symptoms and DSM-IV disorders, substance use disorders, suicidal ideation, and psychiatric hospitalizations.

\section{Results}

\section{Demographic Characteristics of the Study Sample}

Approximately three-quarters ( $\mathrm{N}=735,73.1 \%)$ of the patients identified themselves as of Hispanic origin. Spanish was the primary language for two-thirds of the sample $(\mathrm{N}=$ $660,65.7 \%)$. Approximately seven in $10(\mathrm{~N}=723,71.9 \%)$ were born outside of the mainland United States, most commonly in the Dominican Republic ( $\mathrm{N}=523,52.0 \%)$, Puerto Rico (N=94, 9.4\%), Cuba ( $\mathrm{N}=33,3.3 \%)$, or Ecuador $(\mathrm{N}=12,1.2 \%)$. Study patients were poor and had little formal education. A vast majority ( $\mathrm{N}=867$ of $998,86.9 \%$ ) reported that their total annual family income in 1998 was less than $\$ 12,000$. Most had not completed high school $(\mathrm{N}=612,60.9 \%)$.

\section{Psychotic Symptoms}

Two hundred ten (20.9\%) of the 1,005 patients reported one or more current psychotic symptoms. Among these patients, the most commonly reported psychotic symptoms were auditory hallucinations $(\mathrm{N}=128,61.0 \%)$, a belief that others were spying on or following the patient $(\mathrm{N}=107$, $51.0 \%)$, and visual hallucinations ( $\mathrm{N}=104,49.5 \%)$. Other common symptoms included a belief that people were plotting or trying to poison the patient $(\mathrm{N}=69,32.9 \%)$, the experience of hearing other people's thoughts $(\mathrm{N}=69,32.9 \%)$, thought insertion ( $\mathrm{N}=67,31.9 \%)$, or thought broadcasting $(\mathrm{N}=65,31.0 \%)$. Less commonly, these patients reported a belief that people were secretly testing or experimenting on them $(\mathrm{N}=46$ of $208,22.1 \%)$ and delusions of reference $(\mathrm{N}=47$, $22.4 \%$ ) or control ( $\mathrm{N}=39$ of $209,18.7 \%$ ). The mean number of lifetime psychotic symptoms reported by patients with current psychotic symptoms was $3.5(\mathrm{SD}=2.4)$.

A majority of patients in both study groups were middleaged, female, not currently married, and spoke Spanish as their primary language (Table 1). Compared with patients without psychotic symptoms, those with psychotic symptoms were more likely to be Hispanic $\left(\chi^{2}=4.7, \mathrm{df}=1, \mathrm{p}=0.03\right)$, to be separated or divorced, to have 8 or fewer years of formal education, to have a lower family income, and to report that Spanish was their primary language. There were no other significant group differences in basic sociodemographic characteristics. The rate of psychotic symptoms was $23.7 \%(\mathrm{~N}=104$ of 439$)$ among individuals with annual family incomes below $\$ 6,000$ and $12.1 \%$ ( $N=8$ of 166) for those with annual family incomes of $\$ 18,000$ or more.

\section{Psychotic Symptoms and Mental Disorders}

A substantial proportion of patients with psychotic symptoms met criteria for one of the mental disorders (Table 2). After controlling for the potentially confounding effects of age, sex, race/ethnicity, and primary language, we found that patients with psychotic symptoms were significantly more likely to meet criteria for each of the assessed mental disorders except drug use disorders. Specifically, after we controlled for the demographic covariates, patients with psychotic symptoms were 7.5 times more likely $(95 \%$ confidence interval $[\mathrm{CI}]=4.6-12.0)$ to have panic disorder, 6.6 times more likely $(95 \% \mathrm{CI}=4.5-9.6)$ to have generalized anxiety disorder, 4.9 times more likely (95\% CI=3.4-6.9) to have major depressive disorder, and 3.0 times more likely (95\% CI=1.7-5.1) to have an alcohol use disorder (Table 2).

In the diagnostic substudy, three $(7.1 \%)$ of 42 patients with current psychotic symptoms and three (1.9\%) of 159 patients without current psychotic symptoms according to the screening interview met Composite International Diagnostic Interview DSM-IV criteria for lifetime psychotic disorder ( $p=0.11$, Fisher's exact test). Borderline personality disorder was quite common among the patients with current psychotic symptoms ( $\mathrm{N}=10$ of $47,21.3 \%$ ) and uncommon among those without current psychotic symptoms $(\mathrm{N}=4$ of $171,2.3 \%)\left(\chi^{2}=22.0, \mathrm{df}=1, \mathrm{p}<0.0001\right)$. 
TABLE 1. Sociodemographic Characteristics of 1,005 Adult Primary Care Patients With and Without Current Psychotic Symptoms ${ }^{\text {a }}$

\begin{tabular}{|c|c|c|c|c|c|c|c|}
\hline \multirow[t]{2}{*}{ Characteristic } & \multicolumn{2}{|c|}{$\begin{array}{l}\text { Psychotic Symptoms } \\
\qquad(\mathrm{N}=210)\end{array}$} & \multicolumn{2}{|c|}{$\begin{array}{l}\text { No Psychotic Symptoms } \\
\qquad(\mathrm{N}=795)\end{array}$} & \multicolumn{3}{|c|}{ Analysis } \\
\hline & Mean & SD & Mean & SD & $\mathrm{t}$ & df & $\mathrm{p}$ \\
\hline \multirow[t]{2}{*}{ Age (years) } & 51.8 & 12.4 & 53.5 & 12.1 & 1.8 & 1003 & 0.08 \\
\hline & $\mathrm{N}$ & $\%$ & $\mathrm{~N}$ & $\%$ & $\chi^{2}$ & df & $\mathrm{p}$ \\
\hline Female gender & 162 & 77.1 & 592 & 74.5 & 0.6 & 1 & 0.43 \\
\hline Race/ethnicity & & & & & 5.0 & 2 & 0.08 \\
\hline Hispanic & 166 & 79.0 & 569 & 71.6 & & & \\
\hline Black, non-Hispanic & 37 & 17.6 & 181 & 22.8 & & & \\
\hline White, non-Hispanic & 7 & 3.3 & 45 & 5.7 & & & \\
\hline Marital status & & & & & 11.1 & 3 & 0.01 \\
\hline Married $^{b}$ & 42 & 20.0 & 214 & 26.9 & & & \\
\hline Separated/divorced & 107 & 51.0 & 320 & 40.3 & & & \\
\hline Never married & 44 & 21.0 & 157 & 19.7 & & & \\
\hline Widowed & 17 & 8.1 & 104 & 13.1 & & & \\
\hline Primary language Spanish & 150 & 71.4 & 510 & 64.2 & 3.9 & 1 & 0.48 \\
\hline \multirow[t]{2}{*}{ Immigrant $^{c}$} & 160 & 76.2 & 568 & 71.4 & 1.8 & 1 & 0.17 \\
\hline & $\mathrm{N}$ & $\%$ & N & $\%$ & z & & $\mathrm{p}$ \\
\hline Education (years) & & & & & 2.2 & & 0.03 \\
\hline $0-8$ & 102 & 48.6 & 324 & 40.8 & & & \\
\hline $9-12$ & 75 & 35.7 & 302 & 38.0 & & & \\
\hline$>12$ & 33 & 15.7 & 169 & 21.3 & & & \\
\hline \multicolumn{8}{|c|}{ Annual household income $(\$)^{c}$} \\
\hline$<6,000$ & 104 & 49.5 & 335 & 42.5 & 2.2 & & 0.03 \\
\hline $6,000-11,999$ & 86 & 41.0 & 342 & 43.4 & & & \\
\hline $12,000-17,999$ & 12 & 5.7 & 53 & 6.7 & & & \\
\hline $18,000-35,999$ & 8 & 3.8 & 40 & 5.1 & & & \\
\hline$\geq 36,000$ & 0 & 0.0 & 18 & 2.3 & & & \\
\hline
\end{tabular}

${ }^{a}$ Current psychotic symptoms assessed by Mini International Neuropsychiatric Interview.

${ }^{\mathrm{b}}$ Includes cohabiting.

${ }^{c} \mathrm{~N}=998: 210$ patients with and 788 patients without psychotic symptoms.

\section{Impairment}

Patients with psychotic symptoms reported significantly greater impairment in work, family, and social functioning than patients without psychotic symptoms (Table 2). More than half $(\mathrm{N}=115,55.0 \%)$ of the 209 patients with psychotic symptoms for whom data were available stated that during the past month they had missed 1 or more days of work. Patients with psychotic symptoms were significantly more likely to perceive themselves as having poor emotional health and report marital distress and parent or child problems than were patients without psychotic symptoms.

The mean Sheehan Disability Scale scores of patients with psychotic symptoms were significantly greater than those of patients without psychotic symptoms, indicating greater impairment in social and family function (Table 2). A significant correlation between psychotic symptoms and Sheehan Disability Scale score persisted after we controlled for major depression, panic disorder, generalized anxiety disorder, substance use disorders, and the demographic covariates in a multiple linear regression (partial $\mathrm{r}=0.180, \mathrm{df}=989, \mathrm{p}<0.0001$ ).

\section{Suicidal Ideation}

More patients with psychotic symptoms ( $\mathrm{N}=42,20.0 \%)$ than patients without psychotic symptoms $(\mathrm{N}=29,3.7 \%)$ reported current suicidal ideation $\left(\chi^{2}=67.5, \mathrm{df}=1, \mathrm{p}<0.0001\right)$. After controlling for each of the mental disorders (major depression, panic disorder, generalized anxiety disorder, alcohol use disorder, and drug use disorder) and demographic covariates, we found that psychotic symptoms remained significantly correlated with suicidal ideation (odds ratio= $3.4,95 \% \mathrm{CI}=1.9-6.1)$.

\section{Mental Health Care}

Patients with psychotic symptoms were more likely than patients without psychotic symptoms to have received inpatient and outpatient mental health care (Table 2). In a logistic regression model, psychotic symptoms remained significantly associated with a history of psychiatric hospitalization after we controlled for the mental disorders and demographic characteristics (odds ratio $=2.0,95 \% \mathrm{CI}=1.3-3.1$ ).

Approximately half of the patients with psychotic symptoms $(\mathrm{N}=100,47.6 \%)$ reported having taken a prescribed psychotropic medication within the last month. The most common class of psychotropic medications was antidepressants $(\mathrm{N}=77,77.0 \%)$, followed by hypnotics $(\mathrm{N}=65$, 
TABLE 2. Mental Disorder, Impairment, and Treatment of 1,005 Adult Primary Care Patients With Current Psychotic Symptoms ${ }^{\mathrm{a}}$

\begin{tabular}{|c|c|c|c|c|c|c|}
\hline \multirow[t]{2}{*}{ Characteristic } & \multicolumn{2}{|c|}{$\begin{array}{l}\text { Psychotic Symptoms } \\
\qquad(\mathrm{N}=210)\end{array}$} & \multicolumn{2}{|c|}{$\begin{array}{l}\text { No Psychotic Symptoms } \\
\qquad(\mathrm{N}=795)\end{array}$} & \multicolumn{2}{|c|}{ Analysis } \\
\hline & $\mathrm{N}$ & $\%$ & $\mathrm{~N}$ & $\%$ & $\chi^{2}(\mathrm{df}=1)$ & $\mathrm{p}$ \\
\hline Any mental disorder & 130 & 61.9 & 179 & 22.5 & 121.0 & $<0.0001$ \\
\hline Panic disorder & 52 & 24.8 & 31 & 3.9 & 95.4 & $<0.0001$ \\
\hline Generalized anxiety disorder & 81 & 38.6 & 67 & 8.4 & 120.2 & $<0.0001$ \\
\hline Major depression & 89 & 42.4 & 100 & 12.6 & 96.6 & $<0.0001$ \\
\hline Substance use disorder & 29 & 13.8 & 51 & 6.4 & 12.4 & $<0.0001$ \\
\hline Alcohol use disorder & 27 & 12.9 & 40 & 5.0 & 16.4 & $<0.0001$ \\
\hline \multirow{2}{*}{$\begin{array}{l}\text { Drug use disorder } \\
\text { Impairment }\end{array}$} & 8 & 3.8 & 18 & 2.3 & 1.6 & 0.21 \\
\hline & Mean & SD & Mean & SD & $t(d f=999)$ & $\mathrm{p}$ \\
\hline \multirow[t]{2}{*}{ Total score on Sheehan Disability Scale } & 7.2 & 7.1 & 2.3 & 4.6 & 12.0 & $<0.0001$ \\
\hline & $\mathrm{N}$ & $\%$ & $\mathrm{~N}$ & $\%$ & $\chi^{2}(\mathrm{df}=1)$ & $\mathrm{p}$ \\
\hline Poor emotional health (past month) & 59 & 28.1 & 66 & 8.3 & 59.8 & $<0.0001$ \\
\hline Work loss (past month) & 115 & 55.0 & 282 & 35.6 & 26.1 & $<0.0001$ \\
\hline Marital distress ${ }^{c}$ & 18 & 28.6 & 36 & 13.0 & 9.3 & 0.002 \\
\hline Parent/child distress $^{d}$ & 24 & 13.8 & 31 & 4.6 & 20.4 & $<0.0001$ \\
\hline \multicolumn{7}{|l|}{ Treatment } \\
\hline Psychiatric hospitalization (lifetime) & 53 & 25.2 & 81 & 10.2 & 32.5 & $<0.0001$ \\
\hline \multicolumn{7}{|l|}{ Mental health visit } \\
\hline Lifetime & 136 & 64.8 & 236 & 29.7 & 87.4 & $<0.0001$ \\
\hline Past month & 76 & 36.2 & 105 & 13.2 & 59.4 & $<0.0001$ \\
\hline \multicolumn{7}{|l|}{ Psychotropic medication } \\
\hline Lifetime & 127 & 60.5 & 225 & 28.3 & 75.6 & $<0.0001$ \\
\hline Past month & 100 & 47.6 & 143 & 18.0 & 79.1 & $<0.0001$ \\
\hline
\end{tabular}

a Psychotic symptoms (current) were assessed by the Mini International Neuropsychiatric Interview. Because of missing data, the total number of subjects varies from 1,000 to 1,005.

${ }^{\mathrm{b}}$ Emotional health was based on patient self-perceptions.

c Analysis was limited to patients with a marital partner $(\mathrm{N}=340)$ : 63 patients with and 277 patients without psychotic symptoms.

${ }^{\mathrm{d}}$ Analysis was limited to patients who had children $(\mathrm{N}=851)$ : 174 patients with and 677 patients without psychotic symptoms.

$65.0 \%)$, anxiolytics $(\mathrm{N}=44,44.0 \%)$, antipsychotics $(\mathrm{N}=16$, $16.0 \%)$, and mood stabilizers ( $\mathrm{N}=1,1.0 \%)$.

\section{Psychotic Symptoms and Impairment}

Patients with current psychotic symptoms varied in the number of lifetime psychotic symptoms they reported. Across four measures of impairment, higher numbers of lifetime psychotic symptoms were related to higher rates of poor health (Figure 1 and Figure 2).

\section{Discussion}

We found that approximately one in five adults in an urban general medicine practice that serves a low-income population reported that they were currently experiencing one or more psychotic symptoms, most commonly auditory hallucinations. This is greater than five times the rate (20.9\% versus $3.7 \%$ ) reported from an earlier study (5) of a middle-class sample (Kaiser Permanente, Oakland, Calif.) receiving care in a private prepaid internal medicine group practice that used the same psychosis screening interview.

Primary care patients with psychotic symptoms in both studies were highly impaired in self-reported work, social, and family functioning. Despite the marked difference in prevalence, the clinical profiles of psychotic patients from the two studies were remarkably similar. Similarities were observed in the rates of suicidal ideation $(20.0 \%$ in the current study versus $21.6 \%$ in the Kaiser study), recent work loss $(55.0 \%$ versus $54.1 \%)$, fair to poor emotional health $(71.4 \%$ versus $73.0 \%)$, and mean Sheehan Disability Scale social life item score (3.8 versus 4.1 ). This consistency across studies suggests that the psychotic symptom screening interview identified groups with similar degrees of impairing psychopathology in the two practices. It also casts doubt on the hypothesis that the high rate of endorsed psychotic symptoms in the current study was attributable solely to widespread mischaracterization of normal cultural practices as psychopathology. If the endorsement of psychotic items in the current study was strongly related to sanctioned religious beliefs, we would not expect similar levels of impairment in the two studies.

The risk of psychotic symptoms was inversely related to total family income. In the current study, the rate of psychotic symptoms was $23.7 \%$ among individuals with annual family incomes below $\$ 6,000$ and $12.1 \%$ for those with annual family incomes of $\$ 18,000$ or more. In the predominantly middle-class Kaiser sample, there was also a strong inverse relationship between income and risk of psychotic symptoms; in that study, patients in the lowest income group $(<\$ 15,000 /$ year $)$ were far more likely to re- 
FIGURE 1. Number of Lifetime Psychotic Symptoms in Relation to Impairment and Treatment Among 1,005 Adult Primary Care Patients With and Without Current Psychotic Symptoms

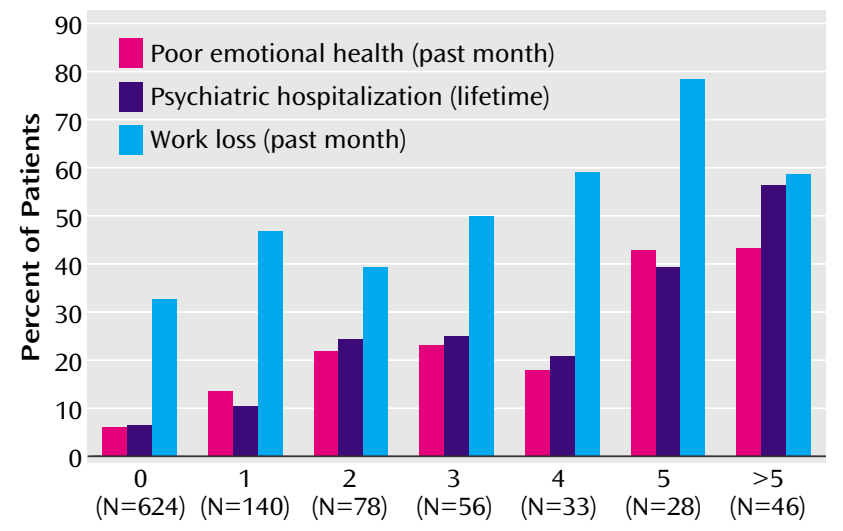

Number of Lifetime Psychotic Symptoms

port psychotic symptoms than patients in the highest income group ( $\geq \$ 40,000 /$ year) ( $12.1 \%$ versus $0.9 \%$ ) (5). In other words, the highest income group of the current study and the lowest income group of the Kaiser study had a similar prevalence of psychotic symptoms. Because findings from both studies are cross-sectional, the results do not address the issue of the causal relationship between psychotic symptoms and poverty.

Patients endorsing psychotic symptoms in the current study were more likely to be Hispanic and to speak Spanish as their primary language. This raises the possibility that cultural factors may also be contributing to the elevated prevalence of psychotic symptoms in this sample. Low-income Caribbean Hispanics tend to report auditory, visual, and tactile misperceptions during depressive and anxiety states and may attribute psychopathology to forms of spiritual influence $(18,23,31)$. The folk labels for these experiences are widely known in Caribbean cultures (e.g., celajes [glimpses], shadows, "hearing your name called"), and their assessment is part of folk healing practices (22). Hispanic patients may reveal these idioms of distress during clinical evaluations and be misdiagnosed with hallucinations or delusions. This was found to be the case in the clinician validation of the National Institute of Mental Health Diagnostic Interview Schedule (DIS) in Puerto Rico $(18,32)$. Lay interviewers using the DIS tended to misdiagnose perceptual alterations and spiritual beliefs as psychotic symptoms. This resulted in an undercount of dysthymic disorder (4.7\% versus $7.5 \%$ ) when it occurred in the context of low-grade depression, since the DIS excludes dysthymia in the presence of psychosis $(18,32)$.

It is difficult to determine the clinical significance of psychotic symptoms per se that are identified through screening interviews, especially in cross-cultural samples. Although data from the diagnostic substudy suggest that few of these patients meet formal criteria for a psychotic disorder, many are nonetheless substantially impaired.
FIGURE 2. Number of Lifetime Psychotic Symptoms in Relation to Score on Sheehan Disability Scale Among 1,005 Adult Primary Care Patients With and Without Current Psychotic Symptoms

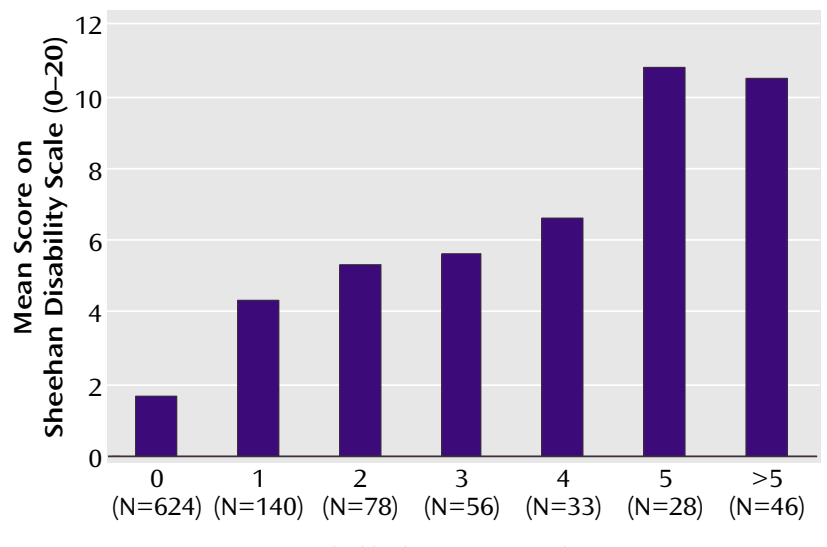

Number of Lifetime Psychotic Symptoms

The risk of impairment tends to increase with an increasing number of reported lifetime psychotic symptoms. However, it is unclear whether the impairment is attributable to the presence of low-grade psychosis in these patients or to a tendency among Hispanic subjects to report certain idioms of distress that have a general association with psychopathology on an instrument intended to capture psychotic symptoms. Both factors may well be contributing independently to the high rate of psychotic symptoms in this sample.

Disentangling these possibilities will require additional clinical and cultural data. It would be useful to have contextual information concerning the timing of symptom onset, the duration and persistence of symptoms, whether or not the symptoms are pervasive, and the extent to which the psychotic symptoms intrude in daily life and adversely influence behavior. In addition, ethnographic data would be required, such as on the community's assessment of the normality of the symptoms, their perceived causation, and their connection to folk healing practices and spiritual beliefs. Other Caribbean idioms of distress should also be assessed, such as ataque de nervios (attack of nerves), a fit of intense emotionality and loss of control precipitated by stressful events. Ataque is nonspecifically associated with mood and anxiety disorders, is often characterized by dissociative symptoms, and has been associated with perceptual distortions in a primary care sample in rural Puerto Rico $(31,33)$.

Rates of panic disorder, generalized anxiety disorder, major depression, alcohol use disorder, and borderline personality disorder were high among the primary care patients with psychotic symptoms in the current study. Primary care physicians, especially those who work in economically disadvantaged areas, should be aware of these associations. Careful clinical assessment will be required to determine in each case the treatment relevance of these symptoms. The detection of psychotic symptoms in pa- 
tients with known nonpsychotic mental disorders may have important implications for treatment. For example, major depression without associated psychotic symptoms often responds to an antidepressant medication alone, but major depression with psychotic symptoms responds more favorably to a combination of antipsychotic and antidepressant medications than to either medication alone (27). However, there is little clinical literature to guide the treatment of low-grade psychotic symptoms, particularly when they occur in the absence of a well-defined mental disorder. The use of the older antipsychotic medications in patients with affective disorders may be associated with a greater risk of tardive dyskinesia (34).

Approximately one-half of the patients with psychotic symptoms reported having taken a psychotropic medication during the past month, and roughly one-third reported having made a mental health visit. These findings raise concerns over unmet needs for mental health care. Whether these symptoms indicate the presence of psychosis or are nonspecific idioms of distress, concern is underscored by the observation that psychotic symptoms were independently related to recent suicidal ideation, Sheehan Disability Scale impairment ratings, and psychiatric hospitalization.

This study has several limitations. First, a substantial percentage $(21 \%)$ of eligible patients who were approached refused to participate in the study. Second, some of the data, particularly concerning psychotropic prescriptions, are of uncertain reliability because they are based on patient self-report. In addition, only limited information is available on the validity of the diagnostic instruments in Spanish-speaking populations with limited formal education (35). Third, no information was available on the rate of posttraumatic stress disorder. This disorder is common in low-income primary care populations (36) and is often complicated with psychotic symptoms $(37,38)$, an association that appears to be higher among Hispanic than nonHispanic clinical samples $(39,40)$. Information was also not available concerning histrionic personality disorder, which may contribute to psychotic response tendencies (41). Fourth, translation of the psychosis screening interview may have introduced subtle inconsistencies in the Spanish and English versions that affected response patterns. However, the interviewers were all bilingual and of Hispanic ancestry. Fifth, information was not available on citizenship status. The experience of being an undocumented migrant might help to explain thoughts of being spied upon. Sixth, the extent to which these findings may be safely generalized to more traditional primary care practices remains unknown.

In conclusion, psychotic symptoms were highly prevalent in this urban general medicine practice, particularly among Hispanic patients. The work, family, and social functioning of patients who reported psychotic symptoms was significantly impaired. Although many patients met criteria for a nonpsychotic mental disorder, few had recog- nized psychotic disorders or received antipsychotic medications. Without additional information, it is premature to conclude that simply endorsing psychotic symptoms during a screening interview defines a mental disorder. Future research should explore the possibility that this response pattern may be affected by Caribbean Hispanic idioms of distress that have a nonspecific association with different psychiatric disorders. However, the remarkably high prevalence of this response pattern and its strong association with suicidal ideation and functional impairment should alert clinicians who work in primary care clinics that serve low-income populations of the need to routinely inquire about the presence of psychotic symptoms.

Received Oct. 12, 2000; revision received Aug. 21, 2001; accepted March 7, 2002. From the College of Physicians and Surgeons of Columbia University and New York State Psychiatric Institute. Address reprint requests to Dr. Olfson, College of Physicians and Surgeons of Columbia University and New York State Psychiatric Institute, 1051 Riverside Dr., New York, NY 10032; mo49@columbia.edu (e-mail).

The authors thank Carlos Blanco, M.D., Ph.D., Renee Goodwin, Ph.D., and Raz Gross, M.D., for their help with the development of this article.

Supported by an investigator-initiated grant from Eli Lilly \& Co., Indianapolis, Ind. PRIME-MD (Patient Health Questionnaire for the Primary Care Evaluation of Mental Disorders) is a trademark of Pfizer Inc., New York, N.Y.

\section{References}

1. Kendler KS, Gallagher TJ, Abelson JM, Kessler RC: Lifetime prevalence, demographic risk factors, and diagnostic validity of nonaffective psychosis assessed in a US community sample. Arch Gen Psychiatry 1988; 53:1022-1031

2. Eaton WW, Romanoski A, Anthony JD, Nestadt G: Screening for psychosis in the general population with a self-report interview. J Nerv Ment Dis 1991; 179:689-693

3. Ross CA, Joshi S: Schneiderian symptoms and childhood trauma in the general population. Compr Psychiatry 1992; 33: 269-273

4. Bromet E, Davies M, Schulz SC: Basic principles of epidemiologic research in schizophrenia, in Handbook of Schizophrenia, vol 3: Nosology, Epidemiology and Genetics. Edited by Tsuang MT, Simpson JC. Amsterdam, Elsevier, 1988, pp 151-168

5. Olfson M, Weissman MM, Leon AC, Farber L, Sheehan DV: Psychotic symptoms in primary care. J Family Practice 1996; 43: 481-488

6. Nichter M: Idioms of distress: alternatives in the expression of psychosocial distress. Cult Med Psychiatry 1981; 5:379-408

7. Kobak KA, Taylor LH, Dottle SL, Greist JH, Jefferson JW, Burroughs D, Katzelnick DJ, Mandell M: A computer administered telephone interview to identify mental disorders in primary care. JAMA 1997; 278:905-910

8. McQuaid JR, Stein MB, Laffaye C, McCahill ME: Depression in a primary care clinic: the prevalence and impact of an unrecognized disorder. J Affect Disord 1999; 55:1-10

9. Olfson M, Broadhead WE, Weissman MM, Leon AC, Farber L, Hoven C, Kathol R: Subthreshold psychiatric symptoms in a primary care practice. Arch Gen Psychiatry 1996; 53:880-886

10. Blacker CVR, Clare AW: The prevalence and treatment of depression in general practice. Psychopharmacology (Berl) 1988; 95(suppl):S14-S17

11. Von Korff M, Shapiro S, Burke JD, Teitlebaum M, Skinner EA, German P, Turner RW, Klein L, Burns B: Anxiety and depression in a primary care clinic: comparison of Diagnostic Interview 
Schedule, General Health Questionnaire, and practitioner assessments. Arch Gen Psychiatry 1987; 44:152-156

12. Dohrenwend BP, Levav I, Shrout P, Schwartz S, Naveh G, Link B, Skodol A, Stueve A: Socioeconomic status and psychiatric disorders: the causation-selection issue. Science 1992; 255:946-952

13. Regier DA, Farmer ME, Rae DS, Myers JK, Kramer M, Robins LN, George LK, Karno M, Locke BZ: One-month prevalence of mental disorders in the United States and sociodemographic characteristics: the Epidemiologic Catchment Area study. Acta Psychiatr Scand 1993; 88:35-47

14. Glover GR, Lesse M, McCrone P: More severe mental illness is concentrated in deprived areas. Br J Psychiatry 1999; 175:544548

15. Marcelis M, Navarro-Mateu F, Murray R, Selten JP, Van Os J: Urbanization and psychosis: a study of 1942-1978 birth cohorts in the Netherlands. Psychol Med 1998; 28:871-879

16. Freeman $\mathrm{H}$ : Schizophrenia and city residence. $\mathrm{Br} J$ Psychiatry Suppl 1994; 23:39-50

17. Lewis G, David A, Andreasson S, Allebeck P: Schizophrenia and city life. Lancet 1992; 340:137-140

18. Guarnaccia PJ, Guevara-Ramos LM, Gonzáles G, Canino GJ, Bird $\mathrm{H}$ : Cross-cultural aspects of psychotic symptoms in Puerto Rico. Res Community Ment Health 1992; 7:99-110

19. Lawson WB, Herrera JM, Costa J: The dexamethasone suppression test as an adjunct in diagnosing depression. J Assoc Acad Minor Phys 1992; 3:17-19

20. Mukherjee S, Shukla S, Woodle J, Rosen AM, Olarte S: Misdiagnosis of schizophrenia in bipolar patients: a multiethnic comparison. Am J Psychiatry 1983; 140:1571-1574

21. Lewis-Fernández R, Kleinman A: Culture, personality, and psychopathology. J Abnorm Psychol 1994; 103:67-71

22. Garrison V: The "Puerto Rican syndrome" in psychiatry and espiritismo, in Case Studies in Spirit Possession. Edited by Crapanzano V, Garrison V. New York, John Wiley \& Sons, 1977, pp 383449

23. Sandoval MC: Santería as a mental health care system: an historical overview. Soc Sci Med 1979; 13B:137-151

24. Janca A, Ustun TB, Sartorius N: New versions of World Health Organization instruments for the assessment of mental disorders. Acta Psychiatr Scand 1994; 90:73-83

25. Maffei C, Fossati A, Agostoni I, Barraco A, Bagnato M, Deborah D, Namia C, Novella L, Petrachi M: Interrater reliability and internal consistency of the Structured Clinical Interview for DSMIV Axis II Personality Disorders (SCID-II), version 2.0. J Personal Disord 1997; 11:279-284

26. Spitzer RL, Kroenke K, Williams JB: Validation and utility of a self-report version of PRIME-MD: the PHQ Primary Care Study. JAMA 1999; 282:1737-1744

27. Sheehan DV, Harnett-Sheehan K, Raj BA: The measurement of disability. Int Clin Psychopharmacol 1996; 11(suppl 3):89-95
28. Leon AC, Shear MK, Portera L, Klerman GL: Assessing impairment in patients with panic disorders: the Sheehan Disability Scale. Soc Psychiatry Psychiatr Epidemiol 1992; 27:78-82

29. Weissman MM, Bothwell S: The assessment of social adjustment by patient self-report. Arch Gen Psychiatry 1976; 33: 1111-1115

30. Sheehan DV, Lecrubier $Y$, Sheehan KH, Amorim P, Janavs J, Weiller E, Hergueta T, Baker R, Dunbar GC: The Mini-International Neuropsychiatric Interview (MINI): the development and validation of a structured diagnostic psychiatric interview for DSM-IV and ICD-10. J Clin Psychiatry 1998; 59(suppl 20):22-33

31. Lewis-Fernández R, Canino C, Ramírez R, Febo V, Bravo M: Latino perspectives on mental health in primary care, in Proceedings of the 49th Institute on Psychiatric Services. Washington, DC, American Psychiatric Association, 1997, number 142

32. Canino GJ, Bird HR, Shrout PE, Rubio-Stipec M, Bravo M, Martínez R, Sesman M, Guevara LM: The prevalence of specific psychiatric disorders in Puerto Rico. Arch Gen Psychiatry 1987; 44: 727-735

33. Guarnaccia PJ, Canino G, Rubio-Stipec M, Bravo M: The prevalence of ataques de nervios in the Puerto Rico Disaster Study. J Nerv Ment Dis 1993; 181:157-165

34. Kane JM: Tardive dyskinesia in affective disorders. J Clin Psychiatry 1999; 60(suppl 5):43-47

35. Villasenor $\mathrm{Y}$, Waitzkin $\mathrm{H}$ : Limitations of a structured psychiatric diagnostic instrument in assessing somatization among Latino patients in primary care. Med Care 1999; 37:637-646

36. Miranda J, Azocar F, Komaromy M, Golding JM: Unmet mental health needs of women in public-sector gynecologic clinics. Am J Obstet Gynecol 1998; 178:212-217

37. Hamner MB, Frueh BC, Ulmer HG, Huber MG, Twomey TJ, Tyson C, Arana GW: Psychotic features in chronic posttraumatic stress disorder and schizophrenia: comparative severity. J Nerv Ment Dis 2000; 188:217-221

38. Hamner MB, Frueh BC, Ulmer HG, Arana GW: Psychotic features and illness severity in noncombat veterans with chronic posttraumatic stress disorder. Biol Psychiatry 1999; 45:846852

39. Mueser KT, Butler RW: Auditory hallucinations in combat-related chronic posttraumatic stress disorder. Am J Psychiatry 1987; 144:299-302

40. Wilcox J, Briones D, Suess L: Auditory hallucinations, posttraumatic stress disorder, and ethnicity. Compr Psychiatry 1991; 32:320-323

41. Folstein MF, Romanoski AJ, Nestadt G, Chahal R, Merchant A, Shapiro S, Kramer M, Anthony J, Gruenberg EM, McHugh PR: Brief report on the clinical reappraisal of the Diagnostic Interview Schedule carried out at the Johns Hopkins site of the Epidemiological Catchment Area program of the NIMH. Psychol Med 1985; 15:809-814 